\title{
QUANTUM TANAKA FORMULA IN TERMS OF QUANTUM BROWNIAN MOTION
}

\author{
YULAN ZHOU ${ }^{凶}$ and CAISHI WANG
}

(Received 6 March 2010)

\begin{abstract}
A quantum local time, which is a generalized operator-valued process, is defined for quantum Brownian motion, and a quantum analogue of the classical Tanaka formula is then established.
\end{abstract}

2010 Mathematics subject classification: primary 81S25.

Keywords and phrases: white noise analysis, quantum local time, quantum Tanaka formula, symbol of generalized operator.

\section{Introduction}

In classical stochastic analysis, we have the following Tanaka formula for Brownian motion $\left\{B_{t}, t \geq 0\right\}$ :

$$
\left|B_{t}-a\right|=|a|+\int_{0}^{t} \operatorname{sgn}\left(B_{s}-a\right) d B_{s}+\int_{0}^{t} \delta_{a}\left(B_{s}\right) d s \quad t>0, a \in \mathbb{R}
$$

where $\int_{0}^{t} \delta_{a}\left(B_{s}\right) d s$ is known as the local time of $\left\{B_{t}, t \geq 0\right\}$ at $a \in \mathbb{R}$. It is proved that the process

$$
\left\{L_{t}^{a}=\int_{0}^{t} \delta_{a}\left(B_{s}\right) d s, t>0, a \in \mathbb{R}\right\}
$$

is the density of the measure $A \mapsto \int_{0}^{t} 1_{A}\left(B_{S}\right) d s$ on $(\mathbb{R}, \mathcal{B}(\mathbb{R}))$, and a continuous Markovian process with respect to $(t, a)$ on $\mathbb{R}_{+} \times \mathbb{R}$; moreover, Chung and Williams [2] applied local time to study the Brownian motion with a rejection wall. Furthermore, the Itô formula was extended to convex functions applying local time.

On the other hand, white noise analysis initiated by Hida [3] is essentially an infinite-dimensional analogue of Schwartz's distribution theory, which has important

The research was supported by the Natural Science Foundation of China (11061032).

(C) 2011 Australian Mathematical Publishing Association Inc. 0004-9727/2011 \$16.00 
applications in many fields, including stochastic analysis and quantum physics [7, 9, 10]. The mathematical framework of white noise analysis is the Gel'fand triple

$$
(E) \subset\left(L^{2}\right) \subset(E)^{*}
$$

over $S(\mathbb{R}) \subset L^{2}(\mathbb{R}) \subset S^{*}(\mathbb{R})$, where $(E)$ and $(E)^{*}$ are known as Hida's testing and generalized functional spaces, respectively. Let $\mathcal{L}=\mathcal{L}\left[(E),(E)^{*}\right]$ be the space of continuous linear operators from $(E)$ to $(E)^{*}$. Elements of $\mathcal{L}$ are called generalized operators (GOs), which are significant generalizations of bounded operators on Hilbert space $\left(L^{2}\right)$.

Quantum Brownian motion, which is an observable of Schwartz class and $\delta$ composable [12, 14], is a very important operator-valued process in white noise analysis. The quantum Itô formula holds only for the product of two quantum semimartingales, hence it is meaningful to investigate the quantum local time and quantum Tanaka formula of quantum Brownian motion. As we will see, the quantum Tanaka formula has a similar form to the classical one.

The paper is organized as follows. In Section 2 we recall some notions, notation and lemmas in white noise analysis. In Section 3 the quantum local time of $\left\{Q_{t} \mid t>0\right\}$ is defined, which is the density of a generalized operator-valued measure (GOVM) from $\mathcal{B}(\mathbb{R})$ to $\mathcal{L}$, and we therefore name it the quantum local time. In the final section, the Tanaka formula is established.

\section{Preliminaries}

In this section, we briefly recall some notions, notation and facts in white noise analysis. For details, see [1, 4-6, 8, 11, 13, 15, 16].

Let $H=L^{2}(\mathbb{R}, d t ; \mathbb{R})$ be the Hilbert space of real-valued square integrable functions on $\mathbb{R}$ with norm $|\cdot|_{0}$ and inner product $\langle\cdot, \cdot\rangle$. The space $E=S(\mathbb{R})$ (respectively $E^{*}=S^{*}(\mathbb{R})$ ) is the space of Schwartz rapidly decreasing (respectively generalized) functionals. $E$ is a nuclear space and we have a Gel'fand triple $E \subset H \subset E^{*}$. The canonical bilinear form on $E^{*} \times E$ denoted by $\langle\cdot, \cdot\rangle$ coincides with the inner product of $H$.

Let $\mu$ be the standard Gaussian measure on $E^{*}$ and let $\left(E^{*}, \mu\right)$ be the white noise space. By the method of second quantization, we have a complex Gel'fand triple,

$$
(E) \subset\left(L^{2}\right) \subset(E)^{*},
$$

which is known as the canonical framework of white noise analysis and $\left(L^{2}\right)=$ $L^{2}\left(E^{*}, \mu ; \mathbb{C}\right)$. The canonical bilinear form on $(E) \times(E)^{*}$ is denoted by $\langle\cdot \cdot, \cdot\rangle$.

Definition 2.1. For $x \in E_{\mathbb{C}}^{*}$, the Wick product of $x$ is defined inductively as follows:

$$
\begin{aligned}
& : x^{\otimes 0}:=1, \\
& : x^{\otimes 1}:=x, \\
& : x^{\otimes n}:=x \hat{\otimes}: x^{\otimes n-1}:-(n-1) \tau \hat{\otimes}: x^{\otimes n-2}: \text { for } n \geq 2
\end{aligned}
$$

where $\tau \in E^{*} \hat{\otimes} E^{*}$ satisfies $\langle\tau, f \otimes g\rangle=\langle f, g\rangle, f, g \in E$. 
For $\xi \in E_{\mathbb{C}}$, the exponential functional $\phi_{\xi}$ associated with $\xi$ is defined as

$$
\phi_{\xi}(x)=e^{\langle x, \xi\rangle-\langle\xi, \xi\rangle / 2}=\sum_{n=0}^{+\infty} \frac{1}{n !}\left\langle: x^{\otimes n}:, \xi^{\otimes n}\right\rangle, \quad x \in E_{\mathbb{C}}^{*}
$$

The set $\left\{\phi_{\xi}, \xi \in E_{\mathbb{C}}^{*}\right\}$ is total in $(E)$. For $T \in \mathcal{L}\left[(E),(E)^{*}\right]$, its symbol $\hat{T}: E_{\mathbb{C}}^{*} \times E_{\mathbb{C}}^{*} \rightarrow$ $\mathbb{C}$ is defined as

$$
\hat{T}(\xi, \eta)=\left\langle T \phi_{\xi}, \phi_{\eta}\right\rangle, \quad \xi, \eta \in E_{\mathbb{C}} .
$$

The self-adjoint operator from $\left(L^{2}\right)$ to $\left(L^{2}\right)$ is called an observable.

LEMma 2.2. Suppose that $\{X(t), t \geq 0\} \subset \mathcal{L}, M \in \mathcal{B}\left(\mathbb{R}_{+}\right)$. If $\{X(t), t \geq 0\}$ satisfies the conditions:

(1) for any $\xi, \eta \in E_{\mathbb{C}}, \widehat{X(\cdot)}(\xi, \eta): M \mapsto \mathbb{C}$ is measurable;

(2) there exist a constant $K, p \geq 0$, and a nonnegative measurable function $c(t)$ in $\mathbb{R}_{+}$integrable in $M$, such that

$$
|\widehat{X(t)}(\xi, \eta)| \leq c(t) \exp \left\{K\left(|\xi|_{p}^{2}+|\eta|_{p}^{2}\right)\right\} \quad \xi, \eta \in E_{\mathbb{C}}
$$

then for any $l, m \in \mathbb{N}$, the integral of quantum white noise of $\{X(t)\}$ in $M$ with respect to $W_{l, m}(d t)$ exists, denoted by

$$
\int_{M} X(t) d W_{l, m}(t)=\int_{M} X(t) \diamond \partial_{t}^{* l} \partial_{t}^{m} d t \in \mathcal{L}
$$

and

$$
\int_{M} X(t) \widehat{d W_{l, m}}(t)(\xi, \eta)=\int_{M} \widehat{X(t)}(\xi, \eta) \xi^{l}(t) \eta^{m}(t) d t \quad \xi, \eta \in E_{\mathbb{C}}
$$

REMARK 2.3. $: W_{l, m}(d t)=\partial_{t}^{* l} \partial_{t}^{m} d t$ is the quantum white noise measure, where $\partial_{t}, \partial_{t}^{*}$ are the annihilation and creation operators.

Definition 2.4. An observable $T$ in $\left(L^{2}\right)$ is called an observable of Schwartz class if, for any $\xi, \eta \in E$, there exists a function $\rho_{\xi, \eta}^{T} \in E_{\mathbb{C}}$ such that

$$
\left\langle P_{T}(S) \phi_{\xi}, \phi_{\eta}\right\rangle=\int_{S} \rho_{\xi, \eta}^{T}(\lambda) d \lambda, \quad S \in \mathcal{B}(\mathbb{R}),
$$

where $P_{T}$ is the spectral measure of $T$. The function $\rho_{\xi, \eta}^{T}$ is called the spectral density of $T$ corresponding to $\xi, \eta$.

LEMMA 2.5. Let $T$ be an observable of Schwartz class with spectral density $\rho_{\xi, \eta}^{T}$. Then for any bounded Borel measurable function $f: \mathbb{R} \rightarrow \mathbb{R}$, we have $f(T) \in \mathcal{L}\left[\left(L^{2}\right)\right]$ and

$$
\widehat{f(T)}(\xi, \eta)=\left\langle f, \rho_{\xi, \eta}^{T}\right\rangle
$$


Proposition 2.6. Let $T$ be an observable of Schwartz class with spectral density $\rho_{\xi, \eta}^{T}$, and let $\Phi_{T}(\xi, \eta)=\rho_{\xi, \eta}^{T}(0)$ for $\xi, \eta \in E_{\mathbb{C}}$. Then $T$ is $\delta$-composable if and only if $\Phi_{T}$ satisfies the following two conditions:

(1) for any $\xi, \xi^{\prime}, \eta, \eta^{\prime} \in E,(s, t) \mapsto \Phi_{T}\left(\xi+s \xi^{\prime}, \eta+t \eta^{\prime}\right), s, t \in \mathbb{R}$, has an entire analytic extension to $\mathbb{C} \times \mathbb{C}$;

(2) there exist constants $C, K, p \geq 0$ such that

$$
\left|\Phi_{T}(\xi, \eta)\right| \leq C \exp \left\{K\left(|\xi|_{p}^{2}+|\eta|_{p}^{2}\right)\right\} \quad \xi, \eta \in E_{\mathbb{C}}
$$

In this case, $\widehat{\delta(T)}(\xi, \eta)=\rho_{\xi, \eta}^{T}(0)$.

Let $(\xi, \eta)_{0}=\langle\xi, \bar{\eta}\rangle$ for $\xi, \eta \in H_{\mathbb{C}}$. The Weyl operator $W(u)$ on $\left(L^{2}\right)$ is defined as

$$
W(u) \phi_{\xi}=\exp \left\{-(\xi, u)_{0}-\frac{|u|_{0}^{2}}{2}\right\} \phi_{\xi+u} .
$$

Obviously $W(u)$ is a unitary operator on $\left(L^{2}\right)$. For $t>0, u_{t}=1_{[0, t]} \in H_{\mathbb{C}}$, let $U_{s}=W\left(i s u_{t}\right), s \in \mathbb{R}$. Then $\left\{U_{s} \mid s \in \mathbb{R}\right\}$ forms a strongly continuous semigroup of unitary operators. By Stone's theorem, there exists a self-adjoint operator $Q_{t}$ such that $W\left(i s u_{t}\right)=e^{i s Q_{t}}$. Then $\left\{Q_{t} \mid t>0\right\}$ is called the quantum Brownian motion, $d Q_{t}=\left(\partial_{t}+\partial_{t}^{*}\right) d t$ and $d O_{t} \cdot d Q_{t}=d t$.

\section{Quantum local time}

In this section, we define the quantum local time of $\left\{Q_{t} \mid t>0\right\}$ and show that it is the density of a measure on the Borel $\sigma$-field $\mathcal{B}(\mathbb{R})$. Let $\delta$ be the Dirac $\delta$-function; then $\delta$ is a Schwartz generalized function.

As the self-adjoint operator, $Q_{t}$ has the spectral decomposition

$$
Q_{t}=\int_{\mathbb{R}} \lambda P_{t}(d \lambda),
$$

where $P_{t}$ is the orthogonal projective operator on $\left(L^{2}\right)$ corresponding to $Q_{t}$ and $U_{s}=\int_{\mathbb{R}} e^{i s \lambda} P_{t}(d \lambda), s \in \mathbb{R}$.

PROPOSITION 3.1. For any $t \geq 0, Q_{t}$ is an observable of Schwartz class with spectral density

$$
\rho_{\xi, \eta}^{t}(\lambda)=\frac{1}{\sqrt{2 \pi t}} \exp \left\{(\xi, \eta)_{0}-\frac{1}{2 t}\left(\lambda-\left(u_{t}, \xi+\eta\right)_{0}\right)^{2}\right\} .
$$

Moreover, $\int_{0}^{t} \delta\left(Q_{s}\right) d s \in \mathcal{L}$ and for any $\xi, \eta \in E_{\mathbb{C}}$,

$$
\int_{0}^{t} \widehat{\delta\left(Q_{s}\right)} d s(\xi, \eta)=\int_{0}^{t} \frac{1}{\sqrt{2 \pi s}} \exp \left\{(\xi, \eta)_{0}-\frac{1}{2 s}\left(u_{s}, \xi+\eta\right)_{0}^{2}\right\} d s .
$$


Proof. Let $\xi, \eta \in E_{\mathbb{C}}$. Then $\left\langle\left\langle P_{t}(\cdot) \phi_{\xi}, \phi_{\eta}\right\rangle: \mathcal{B}(\mathbb{R}) \rightarrow \mathbb{C}\right.$ is a Borel measure. For $s \in \mathbb{R}$,

$$
\begin{aligned}
& \int_{R} e^{i s \lambda}\left\langle\left\langle P_{t}(d \lambda) \phi_{\xi}, \phi_{\eta}\right\rangle=\|\left\langle\int_{R} e^{i s \lambda} P_{t}(d \lambda) \phi_{\xi}, \phi_{\eta} \|=\left\langle W\left(i s u_{t}\right) \phi_{\xi}, \phi_{\eta}\right\rangle\right.\right. \\
& =\exp \left\{-\left(\xi, i s u_{t}\right)_{0}-\frac{\left|i s u_{t}\right|_{0}^{2}}{2}\right\}\left\langle\phi_{\xi+i s u_{t}}, \phi_{\eta}\right\rangle \\
& =\exp \left\{-\left(\xi+\eta, i s u_{t}\right)_{0}-\frac{s^{2} t}{2}+(\xi, \eta)_{0}\right\} \\
& =\int_{R} e^{i s \lambda} \frac{1}{\sqrt{2 \pi t}} \exp \left\{(\xi, \eta)_{0}-\frac{\left(\lambda-\left(u_{t}, \xi+\eta\right)_{0}^{2}\right)}{2 t}\right\} d \lambda
\end{aligned}
$$

which implies

$$
\left\langle P_{t}(d \lambda) \phi_{\xi}, \phi_{\eta}\right\rangle=\frac{1}{\sqrt{2 \pi t}} \exp \left\{(\xi, \eta)_{0}-\frac{\left(\lambda-\left(u_{t}, \xi+\eta\right)_{0}^{2}\right)}{2 t}\right\} d \lambda,
$$

thus, $Q_{t}$ is an observable of Schwartz class with spectral density

$$
\rho_{\xi, \eta}^{t}(\lambda)=\frac{1}{\sqrt{2 \pi t}} \exp \left\{(\xi, \eta)_{0}-\frac{1}{2 t}\left(\lambda-\left(u_{t}, \xi+\eta\right)_{0}\right)^{2}\right\} .
$$

For $\xi, \xi^{\prime}, \eta, \eta^{\prime} \in E$, the function

$$
\begin{aligned}
(s, r) & \mapsto \rho_{\xi+s \xi^{\prime}, \eta+r \eta^{\prime}}^{t}(\lambda) \\
& =\frac{1}{\sqrt{2 \pi t}} \exp \left\{\left(\xi+s \xi^{\prime}, \eta+r \eta^{\prime}\right)_{0}-\frac{1}{2 t}\left(\lambda-\left(u_{t}, \xi+s \xi^{\prime}+\eta+r \eta^{\prime}\right)_{0}\right)^{2}\right\}
\end{aligned}
$$

has an entire analytic extension to $\mathbb{C} \times \mathbb{C}$ and

$$
\left|\rho_{\xi, \eta}^{t}(0)\right| \leq \frac{1}{\sqrt{2 \pi t}} \exp \left\{\frac{3}{2}\left(|\xi|_{p}^{2}+|\eta|_{p}^{2}\right)\right\} .
$$

Hence by Proposition 2.6, for each $t>0, Q_{t}$ is $\delta$-composable, and $\delta\left(Q_{t}\right) \in \mathcal{L}$,

$$
\widehat{\delta\left(Q_{t}\right)}(\xi, \eta)=\left\langle\delta, \rho_{\xi, \eta}^{t}\right\rangle=\rho_{\xi, \eta}^{t}(0)=\frac{1}{\sqrt{2 \pi t}} \exp \left\{(\xi, \eta)_{0}-\frac{1}{2}\left(u_{t}, \xi+\eta\right)_{0}^{2}\right\} .
$$

By Lemma 2.2, $\int_{0}^{t} \delta\left(Q_{s}\right) d s \in \mathcal{L}$ with $c(s)=1 / \sqrt{2 \pi s}, K=1 / 2$ and

$$
\begin{aligned}
\int_{0}^{t} \widehat{\delta\left(Q_{s}\right)} d s(\xi, \eta) & =\int_{0}^{t} \widehat{\delta\left(Q_{s}\right)}(\xi, \eta) d s \\
& =\int_{0}^{t} \frac{1}{\sqrt{2 \pi s}} \exp \left\{(\xi, \eta)_{0}-\frac{1}{2}\left(u_{s}, \xi+\eta\right)_{0}^{2}\right\} d s
\end{aligned}
$$

This concludes the proof. 
Corollary 3.2. For any $a \in \mathbb{R}$ and $t>0$, we have $\int_{0}^{t} \delta_{a}\left(Q_{s}\right) d s \in \mathcal{L}$, and for any $\xi, \eta \in E_{\mathbb{C}}$,

$$
\int_{0}^{t} \widehat{\delta_{a}\left(Q_{s}\right)} d s(\xi, \eta)=\int_{0}^{t} \frac{1}{\sqrt{2 \pi s}} \exp \left\{(\xi, \eta)_{0}-\frac{1}{2 s}\left(a-\left(u_{s}, \xi+\eta\right)_{0}\right)^{2}\right\} d s .
$$

Next, we define a generalized operator valued measure on $(\mathbb{R}, \mathcal{B}(\mathbb{R}))$, with GOvalued density.

Theorem 3.3. For $B \in \mathcal{B}(\mathbb{R}), t>0, \quad \int_{0}^{t} 1_{B}\left(Q_{s}\right) d s \in \mathcal{L} \quad$ and the map $B \mapsto$ $\int_{0}^{t} 1_{B}\left(Q_{s}\right) d s \in \mathcal{L}$ from $\mathcal{B}(\mathbb{R})$ to $\mathcal{L}$ defines a $G O V M$ with density $\int_{0}^{t} \delta_{a}\left(Q_{s}\right) d s$.

Proof. For any $B \in \mathcal{B}(\mathbb{R}), 1_{B}$ is a bounded Borel function, by Lemma $2.5,1_{B}\left(Q_{S}\right) \in$ $\mathcal{L}$ and for $\xi, \eta \in E_{\mathbb{C}}$

$$
\widehat{1_{B}\left(Q_{s}\right)}(\xi, \eta)=\int_{B} \rho_{\xi, \eta}^{s}(\lambda) d \lambda=\int_{B} \frac{1}{\sqrt{2 \pi s}} \exp \left\{(\xi, \eta)_{0}-\frac{\left(\lambda-\left(u_{s}, \xi+\eta\right)_{0}\right)^{2}}{2 s}\right\} d \lambda
$$

which satisfies the conditions in Lemma 2.2, hence $\int_{0}^{t} 1_{B}\left(Q_{s}\right) d s \in \mathcal{L}$ and

$$
\begin{aligned}
\int_{0}^{t} \widehat{1_{B}\left(Q_{s}\right)} d s(\xi, \eta) & =\int_{0}^{t} \widehat{1_{B}\left(Q_{s}\right)}(\xi, \eta) d s \\
& =\int_{0}^{t} \int_{B} \frac{1}{\sqrt{2 \pi s}} \exp \left\{(\xi, \eta)_{0}-\frac{\left(\lambda-\left(u_{s}, \xi+\eta\right)_{0}\right)^{2}}{2 s}\right\} d \lambda d s \\
& =\int_{B} \int_{0}^{t} \frac{1}{\sqrt{2 \pi s}} \exp \left\{(\xi, \eta)_{0}-\frac{\left(\lambda-\left(u_{s}, \xi+\eta\right)_{0}\right)^{2}}{2 s}\right\} d s d \lambda
\end{aligned}
$$

It is easy to see that the complex-valued function $B \mapsto \int_{0}^{t} \widehat{1_{B}\left(Q_{s}\right)} d s(\xi, \eta)$ is $\sigma$-additive on $\mathcal{B}(\mathbb{R})$. In fact, for any $\left\{A_{n}\right\} \subset \mathcal{B}(\mathbb{R}), A_{n} \cap A_{m}=\emptyset$ with $n \neq m$,

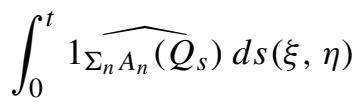

$$
\begin{aligned}
& \left.=\int_{0}^{t}{ }_{\Sigma_{n} A_{n}\left(Q_{s}\right.}\right)(\xi, \eta) d s=\int_{0}^{t} \int_{\Sigma_{n} A_{n}} \rho_{\xi, \eta}^{s}(\lambda) d \lambda d s \\
& =\int_{0}^{t} \int_{\Sigma_{n} A_{n}} \frac{1}{\sqrt{2 \pi s}} \exp \left\{(\xi, \eta)_{0}-\frac{\left(\lambda-\left(u_{s}, \xi+\eta\right)_{0}\right)^{2}}{2 s}\right\} d \lambda d s \\
& =\Sigma_{n} \int_{0}^{t} \int_{A_{n}} \frac{1}{\sqrt{2 \pi s}} \exp \left\{(\xi, \eta)_{0}-\frac{\left(\lambda-\left(u_{s}, \xi+\eta\right)_{0}\right)^{2}}{2 s}\right\} d \lambda d s \\
& \left.=\Sigma_{n} \int_{0}^{t} \widehat{1_{A_{n}}\left(Q_{s}\right)}\right)(\xi, \eta) d s=\Sigma_{n} \int_{0}^{t} \widehat{1_{A_{n}}\left(Q_{s}\right)} d s(\xi, \eta)
\end{aligned}
$$


which shows that $\int_{0}^{t} 1_{(\cdot)}\left(Q_{s}\right) d s$ is a GOVM on $(\mathbb{R}, \mathcal{B}(\mathbb{R}))$, and

$$
\begin{aligned}
\int_{0}^{t} \widehat{1_{B}\left(Q_{s}\right)} d s(\xi, \eta) & =\int_{0}^{t} \widehat{1_{B}\left(Q_{s}\right)}(\xi, \eta) d s \\
& =\int_{0}^{t} \int_{B} \frac{1}{\sqrt{2 \pi s}} \exp \left\{(\xi, \eta)_{0}-\frac{\left(\lambda-\left(u_{s}, \xi+\eta\right)_{0}\right)^{2}}{2 s}\right\} d \lambda d s \\
& =\int_{B} \int_{0}^{t} \frac{1}{\sqrt{2 \pi s}} \exp \left\{(\xi, \eta)_{0}-\frac{\left(\lambda-\left(u_{s}, \xi+\eta\right)_{0}\right)^{2}}{2 s}\right\} d s d \lambda \\
& =\int_{B} \int_{0}^{t} \widehat{\delta_{\lambda}\left(Q_{s}\right)}(\xi, \eta) d s d \lambda=\int_{B} \int_{0}^{t} \widehat{\delta_{\lambda}\left(Q_{s}\right)} d s d \lambda(\xi, \eta),
\end{aligned}
$$

hence

$$
\int_{0}^{t} 1_{B}\left(Q_{s}\right) d s=\int_{B} \int_{0}^{t} \delta_{\lambda}\left(Q_{s}\right) d s d \lambda
$$

and the GOVM $B \mapsto \int_{0}^{t} 1_{B}\left(Q_{s}\right) d s$ has density $\int_{0}^{t} \delta_{\lambda}\left(Q_{s}\right) d s \in \mathcal{L}$.

DEFINITION 3.4. For $t>0$ and $a \in \mathbb{R}$ the generalized operator $\int_{0}^{t} \delta_{a}\left(Q_{s}\right) d s$ is called the quantum local time of quantum Brownian motion $\left\{Q_{t}, t \geq 0\right\}$ at $a \in \mathbb{R}$, denoted by $L_{t}^{a}$. The GO-valued process $\left\{L_{t}^{a} \mid t>0, a \in \mathbb{R}\right\}$ is called the quantum local time process.

Next, we will interpret the meaning of the quantum local time of $L_{t}^{a}$. For any $t \geq 0$, $a \in \mathbb{R}$ and $\xi, \eta \in E_{\mathbb{C}}$

$$
\begin{aligned}
\widehat{L_{t}^{a}}(\xi, \eta) & =\int_{0}^{t} \widehat{\delta_{a}\left(Q_{s}\right)} d s(\xi, \eta) \\
& =\int_{0}^{t} \frac{1}{\sqrt{2 \pi s}} \exp \left\{(\xi, \eta)_{0}-\frac{\left(a-\left(u_{s}, \xi+\eta\right)_{0}\right)^{2}}{2 s}\right\} d s \\
& =\int_{0}^{t} \lim _{\epsilon \rightarrow 0} \frac{1}{2 \epsilon} \int_{a-\epsilon}^{a+\epsilon} \frac{1}{\sqrt{2 \pi s}} \exp \left\{(\xi, \eta)_{0}-\frac{\left(\lambda-\left(u_{s}, \xi+\eta\right)_{0}\right)^{2}}{2 s}\right\} d \lambda d s \\
& =\int_{0}^{t} \lim _{\epsilon \rightarrow 0} \frac{1}{2 \epsilon} \int_{a-\epsilon}^{a+\epsilon} \widehat{\delta_{\lambda}\left(Q_{s}\right)}(\xi, \eta) d \lambda d s \\
& =\int_{0}^{t} \lim _{\epsilon \rightarrow 0} \frac{1}{2 \epsilon} \int_{a-\epsilon}^{a+\epsilon}\left\langle\left\langle\delta_{\lambda}\left(Q_{s}\right) \phi_{\xi}, \phi_{\eta}\right\rangle d \lambda d s\right. \\
& \left.=\int_{0}^{t} \lim _{\epsilon \rightarrow 0} \frac{1}{2 \epsilon} \| \int_{a-\epsilon}^{a+\epsilon} \widehat{\delta_{\lambda}\left(Q_{s}\right)} d \lambda \phi_{\xi}, \phi_{\eta}\right\rangle d s \\
& =\int_{0}^{t} \lim _{\epsilon \rightarrow 0} \frac{1}{2 \epsilon} \int_{a-\epsilon}^{a+\epsilon} \widehat{\delta_{\lambda}\left(Q_{s}\right)} d \lambda(\xi, \eta) d s \\
& =\int_{0}^{t} \lim _{\epsilon \rightarrow 0} \frac{1}{2 \epsilon} 1_{(a-\epsilon, a+\epsilon)}\left(Q_{s}\right) d s(\xi, \eta) .
\end{aligned}
$$


In the sense of GO symbols,

and

$$
L_{t}^{a}=\lim _{\epsilon \rightarrow 0} \frac{1}{2 \epsilon} \int_{0}^{t} 1_{(a-\epsilon, a+\epsilon)}\left(Q_{s}\right) d s
$$

$$
\begin{aligned}
\lim _{\epsilon \rightarrow 0} & \frac{1}{2 \epsilon} \int_{0}^{t} 1_{(a-\epsilon, a+\epsilon)}\left(Q_{s}\right) d s \\
\quad & \lim _{\epsilon \rightarrow 0} \frac{1}{2 \epsilon} L\left\{s ; s \in[0, t], Q_{s}=\lambda I, \lambda \in(a-\epsilon, a+\epsilon)\right\}
\end{aligned}
$$

where $L$ denotes the Lebesgue measure on $\mathbb{R}$.

\section{Quantum Tanaka formula}

In this section the quantum Tanaka formula for the quantum Brownian motion $\left\{Q_{t} \mid t>0\right\}$ is established; its form is similar to the classical case. In this part all quantities are to be interpreted in the sense of GO symbols.

Proposition 4.1. For $t \geq 0, \int_{0}^{t} \operatorname{sgn}\left(Q_{s}\right)\left(\partial_{s}+\partial_{s}^{*}\right) d s \in \mathcal{L}$ and for any $\xi, \eta \in E_{\mathbb{C}}$,

$$
\begin{aligned}
\int_{0}^{t} \operatorname{sgn}\left(Q_{s} \widehat{\left(\partial_{s}+\right.} \partial_{s}^{*}\right) d s(\xi, \eta) \\
=\int_{0}^{t}(\xi(s)+\eta(s)) \int_{\mathbb{R}} \operatorname{sgn}(\lambda) \frac{1}{\sqrt{2 \pi s}} \\
\quad \times \exp \left\{(\xi, \eta)_{0}-\frac{\left(\lambda-\left(u_{s}, \xi+\eta\right)_{0}\right)^{2}}{2 s}\right\} d \lambda d s
\end{aligned}
$$

PROOF. $\operatorname{sgn}(\cdot)$ is bounded on $\mathbb{R}$, by Lemma $2.5, \operatorname{sgn}\left(Q_{s}\right) \in \mathcal{L}$ and for any $\xi, \eta \in E_{\mathbb{C}}$,

$$
\begin{aligned}
\left.\widehat{\operatorname{sgn}\left(Q_{s}\right.}\right)(\xi, \eta) & =\int_{\mathbb{R}} \operatorname{sgn}(\lambda) \rho_{\xi, \eta}^{s}(\lambda) d \lambda \\
& =\int_{\mathbb{R}} \operatorname{sgn}(\lambda) \frac{1}{\sqrt{2 \pi s}} \exp \left\{(\xi, \eta)_{0}-\frac{\left(\lambda-\left(u_{s}, \xi+\eta\right)_{0}\right)^{2}}{2 s}\right\} d \lambda .
\end{aligned}
$$

It is measurable in $s$ and

$$
\left.\mid \widehat{\operatorname{sgn}\left(Q_{s}\right.}\right)(\xi, \eta) \mid \leq \exp \left\{\frac{1}{2}\left(|\xi|_{0}^{2}+|\eta|_{0}^{2}\right)\right\}
$$

by Lemma 2.2, $\operatorname{sgn}\left(Q_{s}\right)$ is integrable in $(0, t]$ with respect to $W_{0,1}(d s)+W_{1,0}(d s)$. Also

$$
\begin{aligned}
& \int_{0}^{t} \operatorname{sgn}\left(Q_{s} \widehat{\left(\partial_{s}+\right.} \partial_{s}^{*}\right) d s(\xi, \eta) \\
& \quad=\int_{0}^{t} \operatorname{sgn}\left(Q_{s} \widehat{\left(\partial_{s}+\right.} \partial_{s}^{*}\right) d s(\xi, \eta) \\
& \quad=\int_{0}^{t} \widehat{\operatorname{sgn}\left(Q_{s}\right)} \partial_{s} d s(\xi, \eta)+\int_{0}^{t} \widehat{\operatorname{sgn}\left(Q_{s}\right)} \partial_{s}^{*} d s(\xi, \eta)
\end{aligned}
$$




$$
\begin{aligned}
= & \left.\left.\int_{0}^{t} \xi(s) \widehat{\operatorname{sgn}\left(Q_{s}\right.}\right)(\xi, \eta) d s+\int_{0}^{t} \eta(s) \widehat{\operatorname{sgn}\left(Q_{s}\right.}\right)(\xi, \eta) d s \\
= & \int_{0}^{t}(\xi(s)+\eta(s)) \int_{\mathbb{R}} \operatorname{sgn}(\lambda) \frac{1}{\sqrt{2 \pi s}} \\
& \times \exp \left\{(\xi, \eta)_{0}-\frac{\left(\lambda-\left(u_{s}, \xi+\eta\right)_{0}\right)^{2}}{2 s}\right\} d \lambda d s .
\end{aligned}
$$

This concludes the proof.

Corollary 4.2. For $t>0, a \in \mathbb{R}$, we have $\int_{0}^{t} \operatorname{sgn}\left(Q_{s}-a I\right)\left(\partial_{s}+\partial_{s}^{*}\right) d s \in \mathcal{L}$ and

$$
\begin{aligned}
\int_{0}^{t} \operatorname{sgn}\left(Q_{s}-\widehat{a I)\left(\partial_{s}\right.}+\partial_{s}^{*}\right) d s(\xi, \eta) \\
=\int_{0}^{t}(\xi(s)+\eta(s)) \int_{\mathbb{R}} \operatorname{sgn}(\lambda-a) \frac{1}{\sqrt{2 \pi s}} \\
\quad \times \exp \left\{(\xi, \eta)_{0}-\frac{\left(\lambda-\left(u_{s}, \xi+\eta\right)_{0}\right)^{2}}{2 s}\right\} d \lambda d s .
\end{aligned}
$$

PROOF. The result follows by a simple computation.

THEOREM 4.3. For any $t>0$,

$$
\left|Q_{t}\right|=\int_{0}^{t} \operatorname{sgn}\left(Q_{s}\right)\left(\partial_{s}+\partial_{s}^{*}\right) d s+\int_{0}^{t} \delta\left(Q_{s}\right) d s .
$$

Proof. By Proposition 3.1, $Q_{t}$ is an observable of Schwartz class:

$$
Q_{t}=\int_{\mathbb{R}} \lambda P_{t}(d \lambda) \quad \text { and } \quad \widehat{Q}_{t}(\xi, \eta)=\int_{\mathbb{R}} \lambda \rho_{\xi, \eta}^{t}(d \lambda), \quad \xi, \eta \in E_{\mathbb{C}} .
$$

Applying Lemma 2.5 for $f(x)=|x|$ we have

$$
\begin{aligned}
\left|\widehat{Q_{t}}\right|(\xi, \eta)= & \int_{\mathbb{R}}|\lambda| \rho_{\xi, \eta}^{t}(\lambda) d \lambda \\
= & \int_{\mathbb{R}}|\lambda| \frac{1}{\sqrt{2 \pi t}} \exp \left\{(\xi, \eta)_{0}-\frac{\left(\lambda-\left(u_{t}, \xi+\eta\right)_{0}\right)^{2}}{2 t}\right\} d \lambda \\
= & \int_{\mathbb{R}}\left|\sqrt{t} \lambda+\left(u_{t}, \xi+\eta\right)_{0}\right| \frac{1}{\sqrt{2 \pi}} \exp \left\{(\xi, \eta)_{0}-\frac{\lambda^{2}}{2}\right\} d \lambda \\
= & \int_{\mathbb{R}} \frac{1}{\sqrt{2 \pi}} \exp \left\{(\xi, \eta)_{0}-\frac{\lambda^{2}}{2}\right\} \\
& \quad \times \int_{0}^{t} \operatorname{sgn}\left(\sqrt{s} \lambda+\left(u_{s}, \xi+\eta\right)_{0}\right)\left(\frac{\lambda}{2 \sqrt{s}}+(\xi(s)+\eta(s))\right) d s d \lambda
\end{aligned}
$$




$$
\begin{aligned}
& =\int_{\mathbb{R}} \int_{0}^{t} \frac{\lambda}{2 \sqrt{2 \pi s}} \operatorname{sgn}\left(\sqrt{s} \lambda+\left(u_{s}, \xi+\eta\right)_{0}\right) \exp \left\{(\xi, \eta)_{0}-\frac{\lambda^{2}}{2}\right\} d s d \lambda \\
& +\int_{\mathbb{R}} \frac{1}{\sqrt{2 \pi}} \exp \left\{(\xi, \eta)_{0}-\frac{\lambda^{2}}{2}\right\} \\
& \times \int_{0}^{t} \operatorname{sgn}\left(\sqrt{s} \lambda+\left(u_{s}, \xi+\eta\right)_{0}\right)(\xi(s)+\eta(s)) d s d \lambda \\
& =-\int_{0}^{t} \frac{1}{2 \sqrt{2 \pi s}} \int_{\mathbb{R}} \operatorname{sgn}\left(\sqrt{s} \lambda+\left(u_{s}, \xi+\eta\right)_{0}\right) d\left(\exp \left\{(\xi, \eta)_{0}-\frac{\lambda^{2}}{2}\right\}\right) d s \\
& +\int_{0}^{t}(\xi(s)+\eta(s)) \int_{\mathbb{R}} \operatorname{sgn}\left(\lambda+\left(u_{s}, \xi+\eta\right)_{0}\right) \frac{1}{\sqrt{2 \pi}} \\
& \times \exp \left\{(\xi, \eta)_{0}-\frac{\lambda^{2}}{2}\right\} d \lambda d s \\
& =\frac{1}{2} \int_{0}^{t} \frac{1}{\sqrt{2 \pi s}} \int_{\mathbb{R}} \exp \left\{(\xi, \eta)_{0}-\frac{\lambda^{2}}{2}\right\} \cdot 2 \delta\left(\sqrt{s} \lambda+\left(u_{s}, \xi+\eta\right)_{0}\right) d \lambda d s \\
& +\int_{0}^{t}(\xi(s)+\eta(s)) \int_{\mathbb{R}} \operatorname{sgn}(\lambda) \frac{1}{\sqrt{2 \pi s}} \\
& \times \exp \left\{(\xi, \eta)_{0}-\frac{\left(\lambda-\left(u_{s}, \xi+\eta\right)_{0}\right)^{2}}{2 s}\right\} d \lambda d s \\
& =\int_{0}^{t} \frac{1}{\sqrt{2 \pi s}} \exp \left\{(\xi, \eta)_{0}-\frac{\left(u_{s}, \xi+\eta\right)_{0}^{2}}{2 s}\right\} d s \\
& +\int_{0}^{t}(\xi(s)+\eta(s))\left\langle\operatorname{sgn}, \rho_{\xi, \eta}^{s}\right\rangle d s \\
& =\int_{0}^{t} \frac{1}{\sqrt{2 \pi s}} \exp \left\{(\xi, \eta)_{0}-\frac{\left(u_{s}, \xi+\eta\right)_{0}^{2}}{2 s}\right\} d s \\
& \left.+\int_{0}^{t}(\xi(s)+\eta(s)) \widehat{\operatorname{sgn}\left(Q_{s}\right.}\right)(\xi, \eta) d s \\
& =\int_{0}^{t} \widehat{\delta\left(Q_{s}\right)} d s(\xi, \eta)+\int_{0}^{t} \operatorname{sgn}\left(Q_{s} \widehat{\left(\partial_{s}+\right.} \partial_{s}^{*}\right) d s(\xi, \eta) \text {. }
\end{aligned}
$$

Hence $\left|Q_{t}\right|=\int_{0}^{t} \operatorname{sgn}\left(Q_{s}\right)\left(\partial_{s}+\partial_{s}^{*}\right) d s+\int_{0}^{t} \delta\left(Q_{s}\right) d s$.

THEOREM 4.4. For any $t>0$ and $a \in \mathbb{R}$,

$$
\left|Q_{t}-a I\right|=|a| I+\int_{0}^{t} \operatorname{sgn}\left(Q_{s}-a I\right)\left(\partial_{s}+\partial_{s}^{*}\right) d s+\int_{0}^{t} \delta_{a}\left(Q_{s}\right) d s
$$

where $I:\left(L^{2}\right) \rightarrow\left(L^{2}\right)$ is the identity operator. 
PROOF. By the spectral decomposition of $Q_{t} \in \mathcal{L}$,

$$
Q_{t}-a I=\int_{\mathbb{R}}(\lambda-a) P_{t}(d \lambda) \quad \text { and } \quad\left(\widehat{Q_{t}-a} I\right)(\xi, \eta)=\int_{\mathbb{R}}(\lambda-a) \rho_{\xi, \eta}^{t}(d \lambda) .
$$

Then

$$
\left|Q_{t}-a I\right|=\int_{\mathbb{R}}|\lambda-a| P_{t}(d \lambda) \quad \text { and } \quad\left|\widehat{Q_{t}-a} I\right|(\xi, \eta)=\int_{\mathbb{R}}|\lambda-a| \rho_{\xi, \eta}^{t}(d \lambda) .
$$

Applying Lemma 2.5 for $f(x)=|x-a|$, and with a similar computation in Theorem 4.3, Corollary 3.2 as well as Corollary 4.2, the equality

$$
\left|Q_{t}-a I\right|=|a| I+\int_{0}^{t} \operatorname{sgn}\left(Q_{s}-a I\right)\left(\partial_{s}+\partial_{s}^{*}\right) d s+\int_{0}^{t} \delta_{a}\left(Q_{s}\right) d s
$$

holds.

DeFinition 4.5. For $t>0$ and $a \in \mathbb{R}$, the equality in Theorem 4.4,

$$
\left|Q_{t}-a I\right|=|a| I+\int_{0}^{t} \operatorname{sgn}\left(Q_{s}-a I\right)\left(\partial_{s}+\partial_{s}^{*}\right) d s+\int_{0}^{t} \delta_{a}\left(Q_{s}\right) d s,
$$

is called the quantum Tanaka formula of $\left\{Q_{t} \mid t>0\right\}$ at $a$.

REMARK 4.6. The last term in the quantum Tanaka formula is the quantum local time $L_{t}^{a}$ defined in Section 3.

COROLlary 4.7. For $t>0$ and $a \in \mathbb{R}$,

$$
\begin{aligned}
& \left(Q_{t}-a I\right)^{+}=\int_{0}^{t} 1_{(a,+\infty)}\left(Q_{s}\right)\left(\partial_{s}+\partial_{s}^{*}\right) d s+\frac{1}{2} L_{t}^{a}, \\
& \left(Q_{t}-a I\right)^{-}=\int_{0}^{t} 1_{(-\infty, a)}\left(Q_{s}\right)\left(\partial_{s}+\partial_{s}^{*}\right) d s+\frac{1}{2} L_{t}^{a} .
\end{aligned}
$$

\section{References}

[1] D. M. Chung, T. S. Chung and U. C. Ji, 'A characterization theorem for operators on white noise functionals', J. Math. Soc. Japan 51 (1999), 437-447.

[2] K. L. Chung and R. J. Williams, Introduction to Stochastic Integration (Birkhäuser, Boston, 1983).

[3] T. Hida, Analysis of Brownian Functionals, Carleton Mathematical Lecture Notes, 13 (Carleton Univesity, Ottawa, 1975).

[4] T. Hida, H.-H. Kuo, J. Potthoff and L. Streit, White Noise. An Infinite Dimensional Calculus, Mathematics and its Applications, 253 (Kluwer, Dordrecht, 1993).

[5] Z. Y. Huang, 'Quantum white noises-white noise approach to quantum stochastic calculus', Nagoya Math. J. 129 (1993), 23-42.

[6] Z. Y. Huang, C. S. Wang and X. J. Wang, 'Quantum cable equations in terms of generalized operators. Recent developments in infinite-dimensional analysis and quantum probability', Acta Appl. Math. 63 (2000), 151-164. 
[7] Z. Y. Huang and J. A. Yan, Introduction to Infinite Dimensional Stochastic Analysis (Kluwer, Dordrecht, 2000).

[8] R. L. Hudson and K. R. Parthasarathy, 'Quantum Itô's formula and stochastic evolutions', Comm. Math. Phys. 93 (1984), 301-323.

[9] N. Obata, White Noise Calculus and Fock Space, Lecture Notes in Mathematics, 1577 (Springer, Berlin, 1994).

[10] K. R. Parthasarathy, An Introduction to Quantum Stochastic Calculus, Monographs in Mathematics, 85 (Birkhäuser, Basel, 1992).

[11] J. Potthoff and L. Streit, 'A characterization of Hida distributions', J. Funct. Anal. 101 (1991), $212-229$.

[12] C. S. Wang, 'A new idea to define the $\delta$-function of an observable in the context of white noise analysis', Infin. Dimens. Anal. Quantum Probab. Relat. Top. 8(4) (2005), 659-668.

[13] C. S. Wang and Z. Y. Huang, 'A filtration of Wick algebra and its application to quantum SDEs', Acta Math. Sin. (Engl. Ser.) 20 (2004), 999-1008.

[14] C. S. Wang, Z. Y. Huang and X. J. Wang, ' $\delta$-function of an operator: a white noise approach', Proc. Amer. Math. Soc. 133 (2005), 891-898.

[15] C. S. Wang, Z. Y. Huang and X. J. Wang, 'Analytic characterization for Hilbert-Schmidt operators on Foch space', Acta Math. Sin. (Engl. Ser.) 21 (2005), 787-796.

[16] C. S. Wang, M. Sh. Qu and J. S. Chen, 'A white noise approach to infinitely divisible distributions on Gel'fand triple', J. Math. Anal. Appl. 315 (2006), 425-435.

\section{YULAN ZHOU, School of Mathematics and Information Science, Northwest Normal University, Lanzhou 730070, PR China e-mail: zhouylw123@163.com}

CAISHI WANG, School of Mathematics and Information Science, Northwest Normal University, Lanzhou 730070, PR China e-mail: wangcs@nwnu.edu.cn 